\title{
ePIXfab - The Silicon Photonics Platform
}

\section{Amit Khanna*, Youssef Drissi, Pieter Dumon, Roel Baets, Philippe Absil ${ }^{1}$}

J. Pozo ${ }^{2}$, D.M.R. Lo Cascio ${ }^{2}$, M. Fournier ${ }^{3}$, J-M. Fedeli ${ }^{3}$, L. Fulbert ${ }^{3}$, L. Zimmermann ${ }^{4}$, B. Tillack ${ }^{4,5}$, T. Aalto ${ }^{6}$, P. O'Brien ${ }^{7}$, D. Deptuck ${ }^{8}$, J. Xu ${ }^{8}$, D. Gale ${ }^{8}$

1. Photonics Research Group, Department of Information Technology, IMEC - Ghent University,

2. TNO, Stieltjesweg $1,2628 \mathrm{CK}$, Delft, The Netherlands

Sint-Pietersnieuwstraat 41, 9000 Gent, Belgium

3. CEA, LETI, Minatec 17 rue des Martyrs, 38054 Grenoble, France

4. IHP GmbH - Innovations for High Performance Microelectronics/Leibniz-Institut für innovative

Mikroelektronik, Im Technologiepark 25, 15236 Frankfurt (Oder), Germany

5. Technische Universitaet Berlin, HFT4, Einsteinufer 25, 10587, Berlin, Germany

6. VTT Technical Research Centre of Finland, Tietotie 3, 02150 Espoo, Finland

7. Photonics Packaging Group,Tyndall National Institute,University College Cork, Ireland

8. CMC Microsystems, Innovation Park, Kingston, Canada K7L 3N6

*corresponding author: Amit Khanna ( $\underline{\text { amit.khanna@imec.be) }}$

Abstract: ePIXfab-The European Silicon Photonics Support Center continues to provide state-of-the-art silicon photonics solutions to academia and industry for prototyping and research. ePIXfab is a consortium of EU research centers providing diverse expertise in the silicon photonics food chain, from training users in designing silicon photonics chips to fiber pigtailed chips. While ePIXfab provides world-wide users access to advanced silicon photonics it also focuses its attention to expanding the silicon photonics infrastructure through a network of design houses, access partners and industrial collaborations.

\section{Introduction}

ePIXfab began operation in 2006 as a collaboration agreement between Imec and CEA-LETI, a result of EU FP6 ePIXnet project [1]. Since then ePIXfab has offered 25 MPW shuttle runs catering to more than 170 designs from over 70 worldwide users. Further, in an effort to support the growing need for silicon photonics design skills ePIXfab has organized 8 one week in-depth silicon photonics courses to educate participants in designing silicon photonic circuits and understanding silicon photonics CMOS fabrication processes. The comprehensive trainings are complimented with various workshops and webinars held throughout the year. Since 2011 ePIXfab has increased focus towards industrial users [2]. To encourage the

Integrated Photonics: Materials, Devices, and Applications II, edited by Jean-Marc Fédéli, Laurent Vivien, Meint K. Smit, Proc. of SPIE Vol. 8767, 87670H · @ 2013 SPIE · CCC code: 0277-786X/13/\$18 · doi: 10.1117/12.2020576 
industrial uptake of advanced silicon photonics technology ePIXfab offers silicon photonics feasibility studies through a broad team of technology experts from within the ePIXfab consortium, which encompasses the entire food chain in silicon photonics. More providers have also started to enable silicon photonics access to users, most notably, OPSIS service which began operations in 2011 [3]. Such developments are also aligned with the mission of ePIXfab, to enable the emergence of a sustainable silicon photonics ecosystem.

\section{2. ePIXfab Infrastructure}

Presently, ePIXfab is a consortium of various research centers coordinated by imec- Ghent University partnership. The other members of the consortium are CEA-LETI (France), IHP (Germany), Tyndall National Institute (Ireland), VTT (Finland), TNO (Netherlands) and CMC Microsystems (Canada). This collaboration is supported by EU FP7 ESSenTIAL project [4]. Further, ePIXfab's collaborations with access partners and design houses is instrumental towards easier access for users of silicon photonics technology.

\section{Access infrastructure}

The access infrastructure of ePIXfab has broadened. Access partners provide silicon photonics design for manufacturability support, accept users designs and are the front support for the user.

Europractice-IC service:

Europractice is the low cost IC prototyping service provider in Europe. In 2011 Europractice incorporated Imec's standard silicon photonics technology to their existing offering. ePIXfab has been instrumental in this transition and continues to operate in close collaboration with Europractice to facilitate knowledge transfer. Further, this partnership has been useful towards silicon photonics technology standardization and sustenance.

In 2013, CEA-LETI will also offer their silicon photonics MPW technology through Europractice.

MOSIS Service:

MOSIS, a provider of low-cost prototyping and small volume production services for custom ICs has been at the forefront of IC access for industry and academia in USA. In 2013, MOSIS and ePIXfab announced their collaboration to provide access to silicon photonics technology to MOSIS' users. At present Imec's silicon photonics technologies and Tyndall National Institute's silicon photonics packaging is available for MOSIS's users.

CMC Microsystems:

CMC Microsystems is Canadian government funded support center for creation and application of microand nano-system knowledge through research and a path to commercialization. CMC Microsystems actively supports access to silicon photonics technologies of ePIXfab to Canadian industry and academic institutes. CMC Microsystems organizes annual silicon photonics training along with University of British Columbia to train PhD students and Canadian companies in silicon photonics. This annual course also provides design training for MPW technologies offered by ePIXfab. 
CETC38:

China Electronic Technology Group Corporation No. 38 Research Institute (CETC38) is a Chinese government funded research institute. In 2013 CETC38 will also provide support to Chinese users for access to silicon photonics technology offered by ePIXfab.

\section{Design Support}

Design houses are a relatively nascent part of the silicon photonics food chain and expected to grow in the coming years. Design houses are an increasingly significant part ePIXfab's network, enabling users with sparse knowledge in silicon photonics to obtain design development through commercial silicon photonics design houses. Today, design support is provided through ePIXfab's consortia, commercial design houses and academic research groups.

Imec, CEA-LETI and IHP:

Fabrication houses such as imec, CEA-LETI and IHP also provide design support to users. This is however on a case-by-case basis and available in the case of bilateral cooperation.

TNO:

TNO, the Dutch technology research center specializes amongst else, in full photonic system design and development for on-field deployment. As part of their expertise TNO provides design support to its users.

VLC Photonics:

VLC Photonics is a commercial design house based in Valencia, Spain. VLC Photonics applies skills of trained designers in generic photonics integration technologies to enable its users take an informed decision about the appropriate technology for their applications. VLC photonics develops custom integrated optics designs for ePIXfab silicon photonics MPW runs and also support its users with optical characterization. ePIXfab supports VLC photonics towards research and prototyping in silicon photonics. ePIXfab collaborates with VLC Photonics through trainings and direct communication to inform the optical design engineers with latest technology developments.

Silicon Photonics Research Groups in Academic Institutions:

In an effort to bridge the gap between industry and silicon photonics technology, research groups in academic environments often extend support. Such groups are at the forefront of silicon photonics research, most notable groups extending support to industry are, IEF-Universite Paris Sud, Optoelectronics Design Group-Eindhoven University of Technology, Nanophotonics Laboratory-University of Washington, Nanophotonics Group-Cornell University and, Photonics Group-Ghent University.

\section{Fabs}

ePIXfab supports Imec, CEA-LETI and IHP's fabrication facilities to provide silicon photonics MPW shuttle runs to users (details below). 
Packaging

Packaging within ePIXfab is supported by Tyndall National Institute for prototyping to low volume production levels.

\section{Services}

\section{MPW Technology}

ePIXfab started the silicon photonics MPW technology offer with Standard Passives technology with the first MPW shuttle run in 2006 (see Figure 1). This platform provided users reliable and repeatable fabrication of integrated optics components for complex system level research realized in 200mm CMOS fabs using 193 $\mathrm{nm}$ DUV process [5]. Various results have been published as a result of world-wide access of this technology. This technology is provided by CEA-LETI, imec and IHP in their periodic MPW runs as published on ePIXfab website.

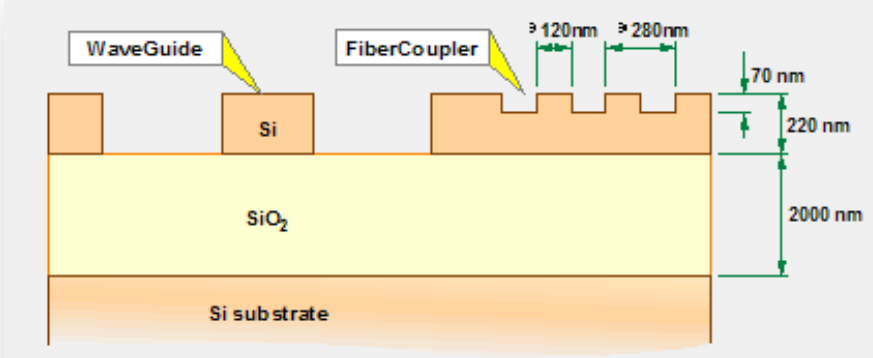

Figure1: Schematic cross-section of the standard passive technology on $220 \mathrm{~nm}$ SOI wafer with 2 etch levels, partial (70nm etch in $220 \mathrm{~nm}$ SOI) and complete etch (220nm etch of $220 \mathrm{~nm}$ SOI).

In 2012 ePIXfab for the first instance offered access to active device technology, CEA-LETI's passive with heater technology. With this technology users could realize tungsten heaters over passive circuits to enable active thermo-optic device tuning. Later in 2012 ePIXfab also offered access to imec's passives with modulator technology and CEA-LETI's passive with photodetector technology, Figure 2. This was complimented with wafer level test results for users and an expanding device library (see section: Design Flow). 


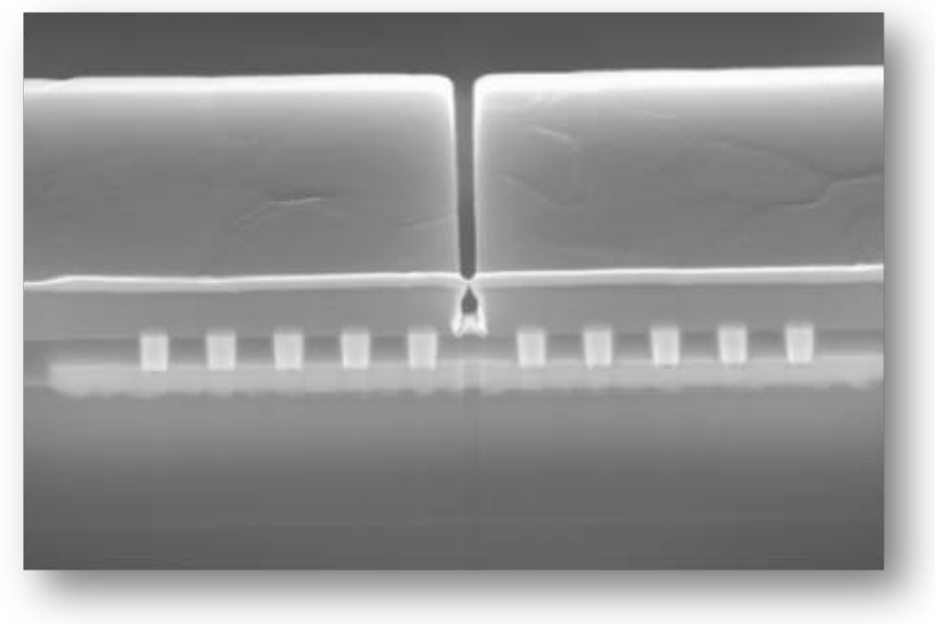

Fugure2: SEM cross-section viewgraph of the Ge-PD fabricated on the CEA-LETI 200mm CMOS line, courtesy CEA-LETI.

In 2013 ePIXfab has announced the availability of full platform in MPW shuttles at both imec and CEA-LETI. Through this technology users can design passive optical circuits with high speed modulators and detectors integrated monolithically on a silicon photonics chip. See Figure 3 for a schematic illustration of such a technology stack at imec.

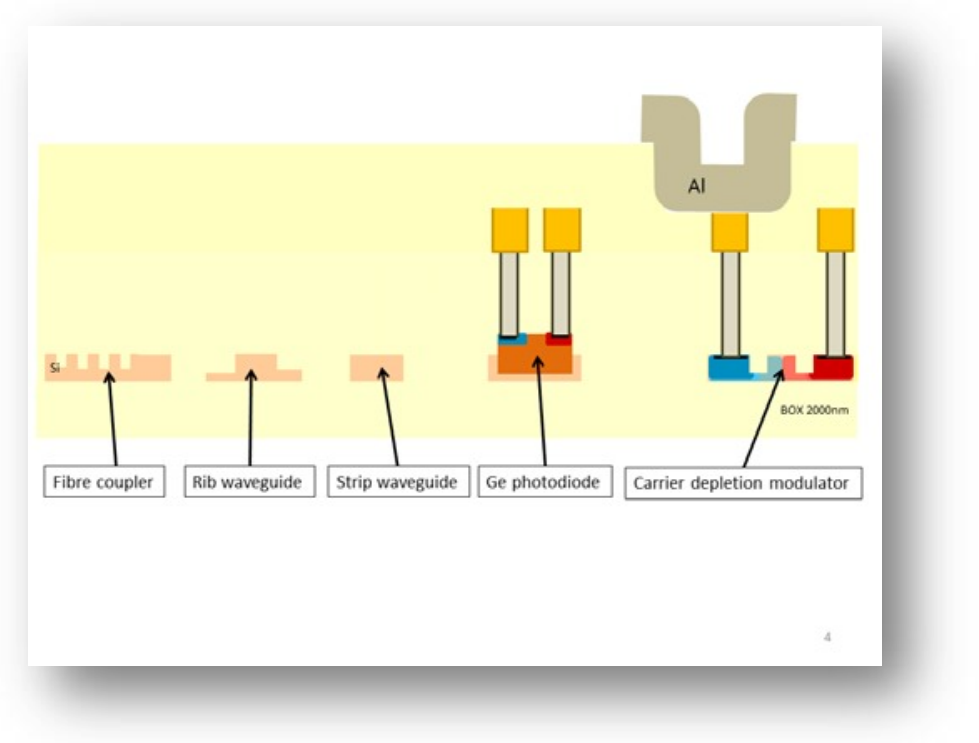

Figure 3: Schematic cartoon of the ISIPP25G fully integrated platform cross-section, courtesy imec. 


\section{Maturity}

Complex silicon photonics technology as shown in Figure 3 is complimented with wafer scale testing. Wafer scale photonic testing enabled with the use of grating couplers has been instrumental in understanding device behavior across wafers and batch-to-batch. This has enabled faster turn-around in device development and functionality driven process improvements.

\section{Design Kits}

After signing the design kit license agreement (DKLA) users can obtain design kits for the aforementioned MPW technologies. The design kit contains technology handbook; an elaborate documentation of process layers and their purpose. Technology handbook also includes performance metrics of devices included in the standard library, this is pivotal for users to predict the performance of standard components, and therefore their complex photonic circuits before participating in the MPW run. Further, the design kit contains layout handbook, describing in detail the rules for layout of designs in GDSII format with details of various CAD layers and their relation to process steps. These documents are complimented by a design rule check file in commercial softwares such as Calibre, TexEDA and Cadence to enable 'design for manufacturability' test (DFM). Thus users may identify fabrication related errors in designs and iterate prior to submission of their design to the foundry, reducing the design development cycle.

To compliment these files user is provided with sample GDSII files of devices in the device library to directly incorporate in user designs. This is further enabled in supported software environments such as PhoeniX software and IPKISS software through technology files developed by the software company and the foundry specifically for this purpose.

\section{Evolving Design Flow}

The design flow mentioned above continues to evolve. In a supported commercial software environment recommended by the fab, users can follow two routes to incorporate designs into their layouts. These two routes are shown in Figure 4. 


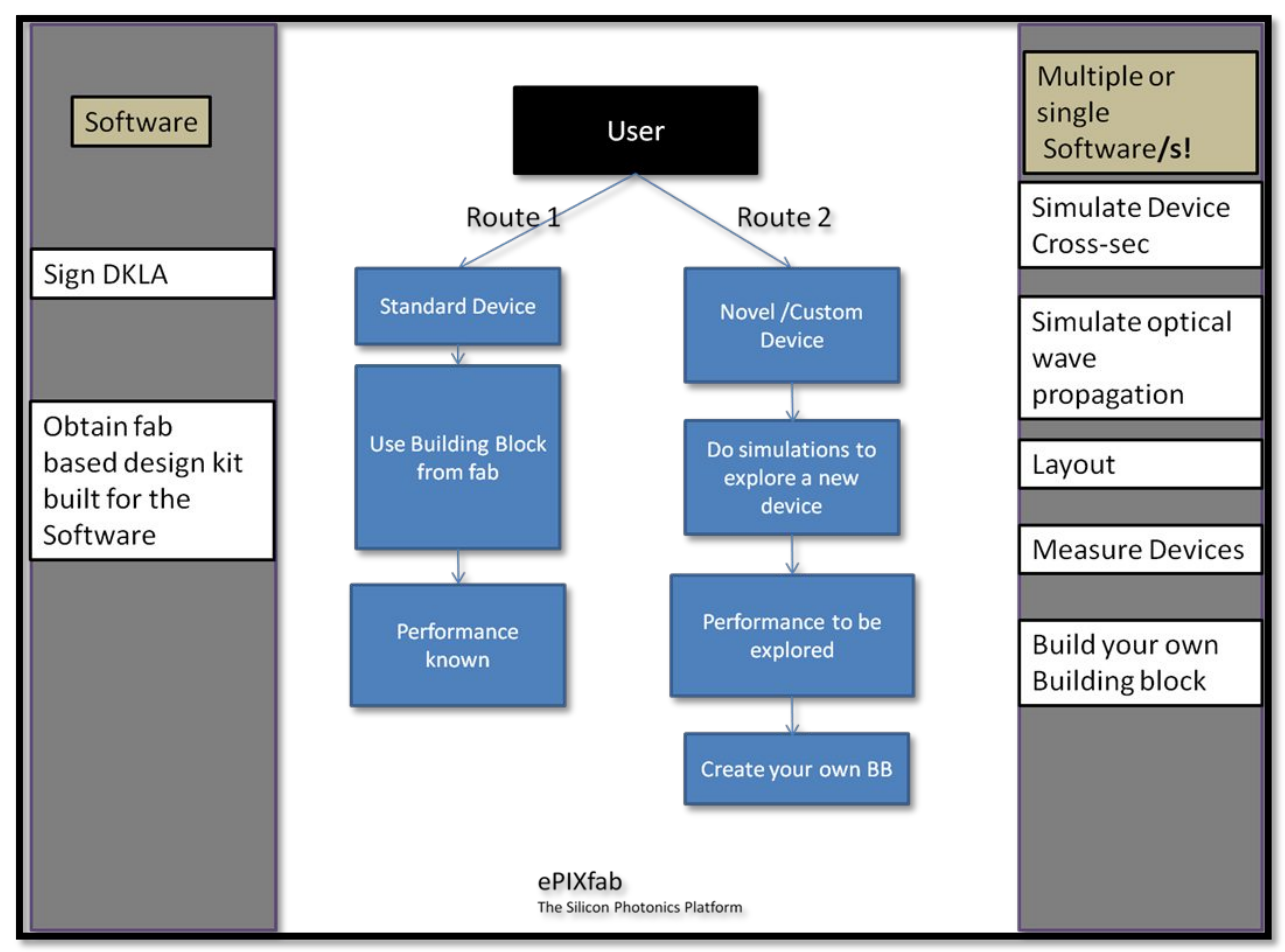

Figure 4: Illustration of the two user routes followed by silicon photonics design engineers. Extract from ePIXfab silicon photonics training, Eindhoven March 2013.

Route 1: enables user to import design library components or building blocks supplied by the fab. These components are tested by the fab and their functional performance is documented in the technology handbooks of the design kit. Further, these components can be imported in circuit simulation tools wherein corresponding s-matrix definitions are pre-defined. Route1 shown in Figure 4 is the preferred route for users to

- Test silicon photonics technology

- Build complex circuit layouts with limited unknowns

- Shorten R\&D/prototyping time

Route 2: Route 2 enables custom device development. The user must begin with electro-magnetic simulations of the device cross-section and study mode field distributions. Typically cross-sectional simulations are performed using FMM, FEM and FDM methods are used depending upon simulation time, device cross-section and level of accuracy required [6]. Cross-sectional simulations are followed by circuit simulation or electro-magnetic field propagation simulations through FDTD simulations or BPM simulations. Other methods of circuit simulations which combine time domain and frequency domain analysis are also available to users. After circuit simulations users are strongly encouraged to analyze fabrication tolerance of their devices and circuits. It may not be necessary that the global functional optimum is the device corresponding to highest fabrication yield. Any such trade-offs must be comprehended by the designer. 
Typically users follow a combination of Route 1 and 2 to realize their custom chips. There is increasing effort from the fab side to grow the library of standard components so users may be provided most commonly used components through the design library via Route 1. In this regard ongoing efforts of the commercial design houses and access providers are also noteworthy as they continue to build their own more complex design libraries.

More efforts across Europe are ongoing to improve the state of the flow through EU FP7 initiatives such as ESSenTIAL and PLAT4M projects. Reader is referred to the project sites to update themselves with the latest developments [4,7].

\section{Packaging \& Integration}

ePIXfab's public packaging and integration roadmap was developed with the support of EU FP7 ESSenTIAL project. The roadmap is available for download on ePIXfab's website [2]. The roadmap incorporates the internal photonics packaging development vision of ePIXfab's partner-Tyndall National Institute, user and access partner feedback on expected market needs in the short $(<1$ year) and medium term (3-5 years) also shown in Table 1. In September 2012 ePIXfab also announced its first cost-shared packaging offer. Designers can now follow pre-defined design rules to enable their designs for packaging with 8 fiber-array-in and 8fiber-array out. These rules are available for viewing on ePIXfab's packaging website. An image of the fiber array packaged over 8 grating couplers is shown in Figure 5.

Table1: ePIXfab packaging roadmap shown above, released in Sep 2012, available on ePIXfab website http://epixfab.eu/

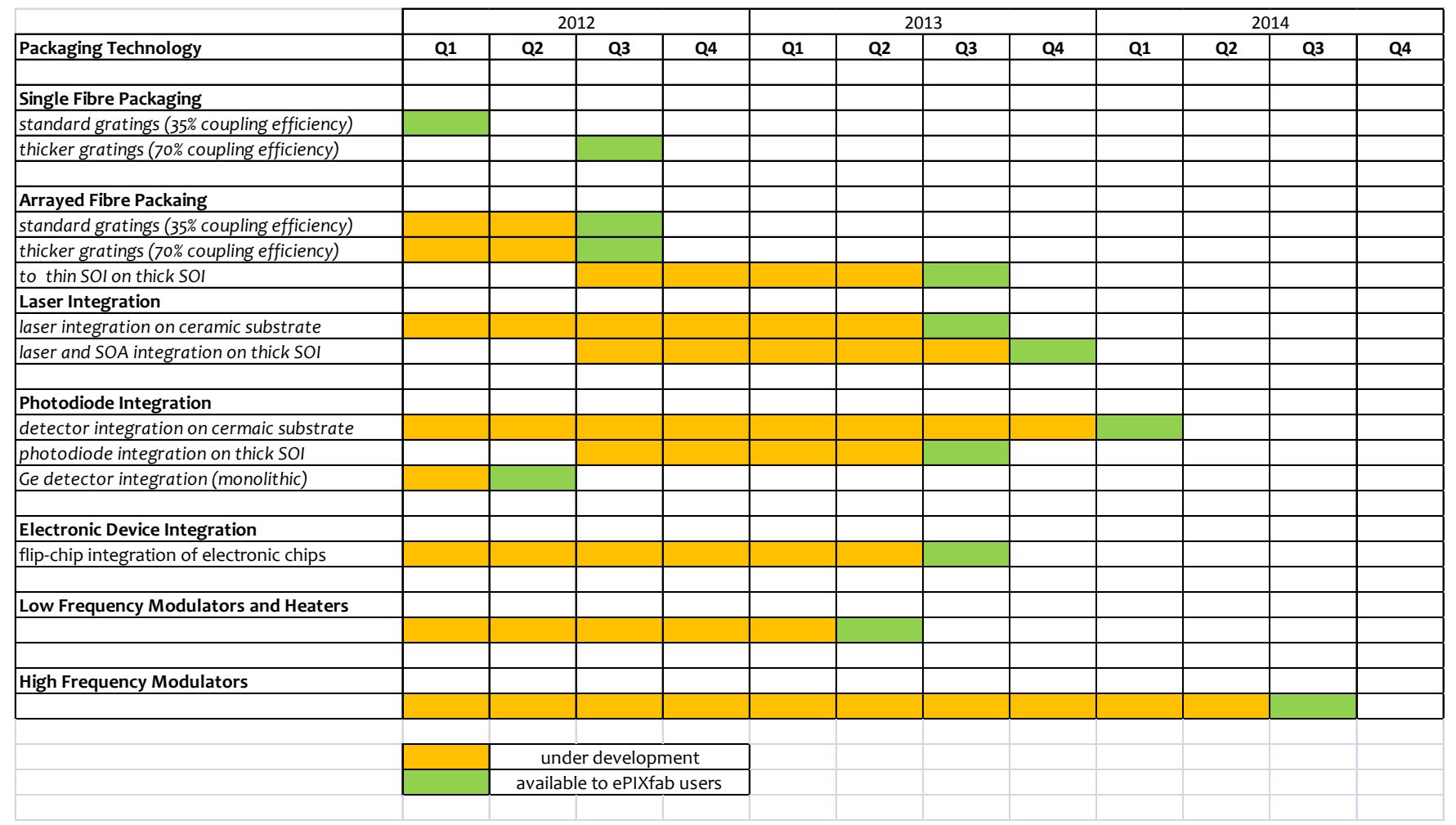




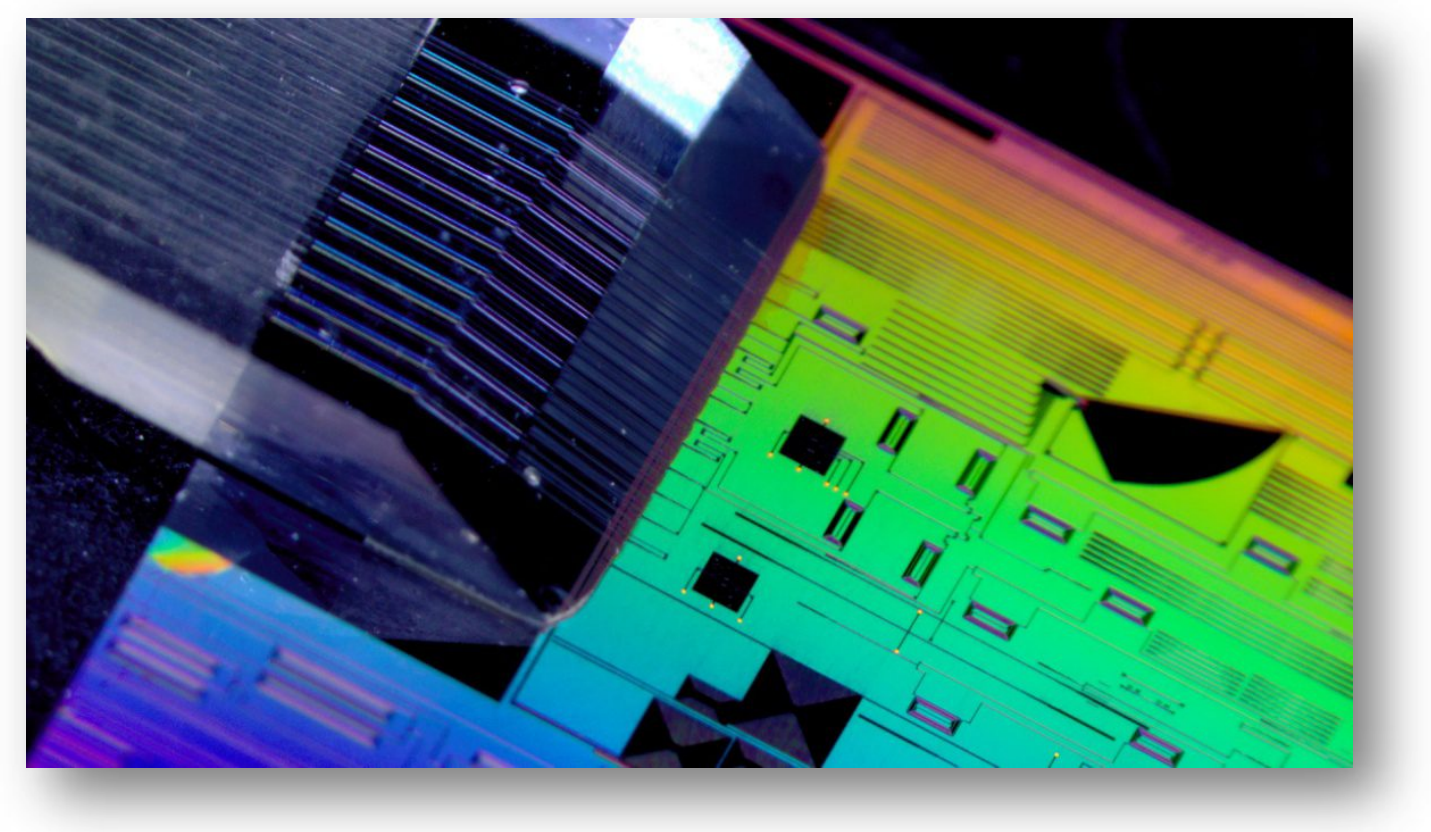

Figure 5: Eight fiber array attached to a passive silicon photonic chip. Image and packaging courtesy Tyndall National Institute, silicon photonic chip manufactured at imec.

\section{Education and Trainings}

Bi-annual one week trainings of ePIXfab have been pivotal towards training of silicon photonics design engineers, these training provide researchers and engineers a great method to learn designing into silicon photonics technology from design and software experts. The training also gives detailed information about CMOS fabrication, packaging and applications of silicon photonics. Annual workshops and webinars are regularly organized to attract industry and users from outside Europe.

\section{Industry Support}

ESSenTIAL (사Xfab services specially targeting industrial uptake of advanced silicon photonics) is an EU FP7 project which provides EU industry and specially SMEs free feasibility studies through ePIXfab's consortium of expertise. The model has been attractive and numerous EU SMEs and industry are exploring the use of silicon photonics technology.

\section{Conclusion}

With the release of more advanced technology such as full integrated platforms at CEA-LETI and imec, and an expanding device library ePIXfab provides state-of-the-art silicon photonics MPW shuttle runs. Further with IHP providing its fabrication facilities for silicon photonics MPW shuttles and Tyndall National Institute developing novel silicon photonics packaging concepts; ePIXfab has approaches a more complete silicon photonics food chain. Various other collaborations with design houses and access partners facilitate easier access to ePIXfab's silicon photonics technology and induce standardization. Together with an increased 
focus on the industry these recent developments provide ePIXfab's community, an unprecedented level of silicon photonics technology maturity at affordable costs for research and prototyping.

\section{References}

[1] ePIXnet website: http://www.epixnet.org/index.php?id=57

[2] ePlxfab website: http://epixfab.eu/

[3] OPSIS website: http://opsisfoundry.org/

[4] ESSenTIAL Project website: http://epixfab.eu/essential-project/essential-project

[5] S. Selvaraja, W. Bogaerts, D. Van Thourhout, R. Baets, Fabrication of Uniform Photonic Devices Using 193nm Optical Lithography in Silicon-on-Insulator ,14th European Conference on Integrated Optics (ECIO), Netherlands, (2008)

[6] A. Khanna, A. Säynätjoki, A. Tervonen and S. Honkanen, "Control of optical mode properties in crossslot waveguides", Applied Optics 48, 34, pp. 6547-6552 (2009)

[7] PLAT4M project website:

http://cordis.europa.eu/fetch?CALLER=ICT UNIFIEDSRCH\&ACTION=D\&DOC=2\&CAT=PROJ\&QUERY= 013da1f1c26a:1ddb:25fddf7c\&RCN=104781 